\title{
PODA PARA LA PRODUCCIÓN DE MADERA DE CALIDAD EN PLANTACIONES DE ÁLAMOS (Populus spp.) EN PATAGONIA NORTE DE ARGENTINA
}

\author{
Davel, M. M. ${ }^{3}$ y Arquero, D. E. ${ }^{4}$
}

\section{RESUMEN}

En el valle del río Negro la producción forestal está íntimamente ligada a la fruticultura, con la madera de álamo se fabrican cajones y bines donde frutas y jugos se envían al mercado local e internacional. Esto hace que la actividad forestal siga los vaivenes de la fruticultura. En los últimos tiempos, para evitar esto, se está pensando en productos alternativos como madera para mueblería y viviendas, entre otros y, para esto, es necesario producir madera de calidad.

Una alternativa incipiente y con potencial, para esta producción en la zona, son los sistemas silvopastoriles. Para producir madera de calidad, estas plantaciones deben ser manejadas desde sus primeras etapas, siendo la poda la principal actividad. En el presente trabajo se estudió el efecto de la aplicación de diferentes intensidades de poda sobre el crecimiento de los árboles y la formación de brotes epicórmicos y se determinaron los momentos oportunos de poda para lograr un diámetro del cilindro con defectos (DCD) constante a lo largo de todo el fuste podado.

Se instalaron dos ensayos de intensidad de poda, uno en la localidad de Añelo

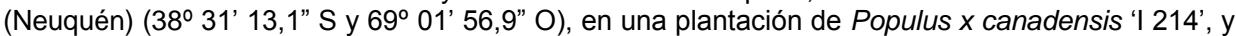
otro en Lamarque (Río Negro) (3930'28,4" S y $65^{\circ} 41^{\prime}$ '59,4" O), donde la especie plantada es Populus $x$ canadensis 'Conti 12', ambas tenían 3 años de edad al momento de la instalación.

Los tratamientos aplicados consistieron en las siguientes intensidades de poda: Poda fuerte (dejando un $25 \%$ de copa verde); Poda intermedia (dejando un $50 \%$ de copa verde); Poda suave (dejando un $75 \%$ de copa verde) y Testigo (sin poda, $100 \%$ de copa verde). Para evaluar diferencias entre tratamientos se realizó un análisis de varianza y para la comparación entre medias de tratamientos, el Test de Tukey.

En cuanto a la intensidad de poda, no se observaron diferencias en el crecimiento en altura en los cuatro años de evaluación. Si hubo diferencias en el crecimiento en diámetro, durante los primeros tres años, entre la poda fuerte y el testigo en 'I 214' y entre la poda fuerte y, la poda suave y el testigo, en 'Conti 12'. Estas diferencias no se observaron al cuarto año.

En cuanto al número de brotes hubo un incremento a medida que aumenta la intensidad de poda, observándose diferencias, en los dos primeros años, que desaparecen al tercer año.

Para determinar el momento oportuno de realizar cada levante, además de la información de los ensayos, se instalaron 12 parcelas permanentes cubriendo diferentes condiciones de sitio y clones presentes en el área. Con la información obtenida se ajustaron modelos que permiten estimar el diámetro en la base de la copa viva a partir de otras variables de más fácil medición como el DAP en la primera poda y el DAP y la altura de poda en los levantes siguientes. Estos modelos fueron validados mediante medidas de error (REMC) y sesgo (DIFA).

En base a los resultados obtenidos se recomienda aplicar podas entre fuertes e intermedias, es decir, dejando entre el 25 y $50 \%$ de copa viva. Podar más suave implica: un mayor número de entradas al monte para alcanzar la misma altura de poda; un aumento importante en el DCD con los distintos levantes de poda; un incremento en el grosor de las ramas y similares crecimientos en diámetro y altura de los árboles que en las podas más fuertes. En base a los

\footnotetext{
${ }^{3}$ MSc. Ingeniero Forestal. CIEFAP, Esquel, Argentina. mdavel@ciefap.org.ar

${ }^{4}$ Ingeniero Forestal. CIEFAP, Esquel, Argentina. darquero@ciefap.org.ar
} 
modelos ajustados, la primera poda se debe realizar cuando los árboles más grandes del rodal alcanzan los $5 \mathrm{~cm}$ de DAP y, los levantes siguientes, cuando el diámetro en la base de la copa viva, también de los árboles más grandes, llegue a los $8-9 \mathrm{~cm}$, de esta forma se obtendría un DCD constante de entre 12 y $14 \mathrm{~cm}$ a lo largo de todo el fuste podado.

Palabras clave: Populus $x$ canadensis 'I 214', Populus $x$ canadensis 'Conti 12', Severidad de podas, Madera libre de nudos, Patagonia.

\section{SUMMARY}

In the Black River Valley the forest production is intimately linked to fruits production; Poplar wood crates for fruits and juices that are sent to the local and international market. This is the reason why forestry follows high and low fruit production. To avoid this, alternative products, as furniture and houses, among others, are analysed and for this kind of purposes clear wood has to be produced.

An alternative emerging and potential management to face that objective in the area is the use of silvopastoral systems. To produce clear wood the plantations are to be managed from its early stages, being pruning the main practice. In this work, the effect of different pruning severities application on trees growth and adventitious (epicormic) shoots formation was studied, as well as the appropriate time of pruning to obtain a constant cylinder with defects (knotty core) diameter.

To achieve the first objective two pruning severity trials were installed; one at Añelo

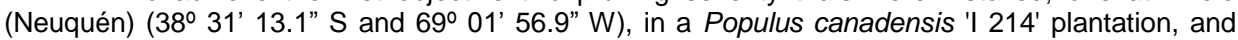
another at Lamarque (Río Negro) (393' 28.4" S and 6541'59.4" W), where planted species is Populus canadensis 'Conti 12'. Both plantations were 3 years old at the trials establishment.

Pruning severities applied were heavy (leaving 25\% green crown), intermediate (leaving $50 \%$ green crown), light (leaving $75 \%$ green crown) and control trees (no pruning, $100 \%$ green crown). To evaluate differences between treatments a variance analysis was performed and to compare treatment means a Tukey test was applied.

Regarding to pruning severity, there were no differences in height growth over the four measurement years. There were differences in the diameter growth, during the first three years, between heavy pruning and control in 'I 214' and between heavy pruning, light pruning and the control, in 'Conti 12'. These differences were not observed in the fourth year. Regarding to the adventitious shoots number there was an increase when the pruning severity is stronger, with differences in the first two years, which disappear in the third year.

To determine the lifting times, information from the trials was used and also 12 permanent plots covering different conditions of sites and clones present in the area were established. Models were adjusted to allow the estimation of the diameter at the base of the green cup based on other variables of easier measurement, as the $\mathrm{DBH}$ in the first pruning and $\mathrm{DBH}$ and height of the following lifts. These models were validated using measures of error and bias.

Based on the obtained results it is recommended to apply pruning between heavy and intermediate, leaving $25 \%$ to $50 \%$ of green crown. A lower severity implies a greater number of entries to the forest to obtain the same pruning height; a significant increase in the knotty core with the different pruning lifts; and an increase in the diameter of the branches and similar growth in diameter and height. On the basis of the obtained models, the first pruning lift should be done when the largest trees in the stand have a $\mathrm{DBH}$ of $5 \mathrm{~cm}$, and the following pruning lifts, when diameter at the base of the green cup has 8-9 cm. That way, a constant DCD between 12 and $14 \mathrm{~cm}$ would be obtained.

Key words: Palabras clave: Populus $x$ canadensis 'I 214', Populus x canadensis 'Conti 12', Pruning severity, Clear wood, Patagonia. 


\section{INTRODUCCIÓN}

En la Patagonia argentina las salicáceas son una de las principales productoras de madera dentro de las especies implantadas (Valor Agregado, 2005). En el valle del Río Negro es donde se concentra la mayor superficie forestada con esta especie, con alrededor de 2.800 ha en macizos y $12.150 \mathrm{~km}$ de cortinas forestales (Serventi, 2011).

El consumo anual de madera por parte de la industria en el Valle del Río Negro fue en las últimas décadas de 150.000 a 300.000 t/año y el complejo forestal e industrial existente ocupa unos 2.800 empleados permanentes y alrededor de 1.500 temporarios (Serventi y García 2004). En la última década, por problemas de pérdida de competitividad generada por el deterioro del tipo de cambio y la caída permanente de la calidad de la materia prima (madera rolliza), el consumo ha descendido. Otro factor a tener en cuenta, en esta zona, es que la producción forestal está íntimamente ligada a la fruticultura. Con la madera de álamo se fabrican cajones y bines para el envío de frutas y jugos al mercado local y al internacional. La madera producida actualmente se destina en un $50 \%$ de su producción a este fin y, en mucha menor medida, para la fabricación de muebles y materiales para la construcción (García y Serventi, 2012). Esto hace que la actividad forestal siga los vaivenes de la fruticultura. En los últimos tiempos, para evitar esto, se está pensando en productos alternativos, como madera para mueblería y viviendas, entre otros y, para esto, es necesario producir madera de calidad.

Actualmente la mayor parte de la madera producida proviene de cortinas cortaviento sin ningún tipo de manejo. Una alternativa incipiente y con potencial, para la producción de madera de calidad en la zona, son los sistemas silvopastoriles. Serventi (2011) menciona que los cambios y las modificaciones que se están generando en la producción agropecuaria argentina posibilitan la incorporación de nuevas regiones a la actividad productiva. Una situación específica es el caso de la producción ganadera que se ha visto desplazada a zonas que históricamente han sido consideradas como marginales. La región patagónica norte forma parte de este tipo de zonas y tiene gran potencial para asociar la producción de madera de calidad con la ganadería en sistemas silvopastoriles. Mientras se va generando madera de calidad, con turnos que se estiman en $12-15$ años, se va produciendo en la misma superficie forraje más carne, generando ingresos anuales que permiten mejorar la rentabilidad. La producción de 80 a $100-150 \mathrm{~kg} / \mathrm{ha} / \mathrm{año}$ de carne, representa un ingreso muy importante que no se puede dejar de considerar (Serventi, 2011).

Para la producción de madera de calidad, las plantaciones deben ser adecuadamente manejadas desde sus primeras etapas y una de las actividades principales, dentro del manejo, es la poda. Esta tarea, en su aplicación, abarca numerosos temas a considerar, entre los que se encuentran: oportunidad, intensidad y número de levantes y sus efectos sobre el crecimiento y sobre la principal variable indicadora de la calidad del tratamiento, como es el diámetro del cilindro con defectos (DCD).

No existen en la región estudios de poda para estas especies. Casaubon (2004), en el Delta del Paraná, indica que la poda se comienza en general cuando el fuste alcanza los 6 o $7 \mathrm{~cm}$ de diámetro a la altura del pecho (DAP).

Shock et al. (2000) muestran resultados de un ensayo de poda en álamos híbridos en Oregón, USA. Los tratamientos consistieron en podas de hasta 2,7 m, 3,6 m y 4,5 m de altura y levantes posteriores de 0,9 m/año hasta los $5,5 \mathrm{~m}$. Se incluyó también un testigo donde los árboles solo se podaron a $1,8 \mathrm{~m}$. Estos tratamientos implicaron una severidad de poda inicial de entre 22 y $47 \%$ de la altura total de los árboles. En ninguno de los casos se produjo una reducción en el crecimiento.

En coníferas existen muchísimos trabajos que analizan número de levantes e intensidades de poda (Sutton y Crowe, 1975; Knowles et al., 1987; Meneses y Velazco,1992; Rivera y Sobarzo, 1992; Kurtz y Ferruchi, 2000; Todoroki, 2003; Costas et al., 2003; Davel, 2005). Entre los principales resultados generales para las distintas especies surgen los siguientes: 
- $\quad$ El diámetro del cilindro con defectos (DCD) y el tamaño de las ramas decrecen al aumentar la severidad y el número de levantes.

- Un incremento pequeño en el DCD debe ser compensado por un incremento de aproximadamente el doble del DAP para producir la misma cantidad de madera libre de nudos.

- Podas más severas que la remoción del 40 - 50\% de copa viva producen un efecto negativo importante sobre el crecimiento.

- $\quad$ El número de brotes adventicios o epicórmicos aumenta con la intensidad y frecuencia de las podas y este efecto se ve potenciado con la aplicación de raleos.

- Hay que poner énfasis en obtener un pequeño DCD y no la compensación de un DCD mayor con un turno más largo. Al ser pequeño el DCD y más grande el diámetro final de las trozas, más valiosas serán estas.

- La primera poda es determinante del resultado final. Cuanto menos se pode, más rápido se va a tener que realizar el segundo levante y, por otro lado, cuanto más se pode más se va a ver afectado el crecimiento.

La primera poda tiene importancia fundamental en la definición de la oportunidad de los levantes siguientes. En una poda bien realizada, el diámetro máximo sobre muñón (DMSM) de un determinado levante, debe ser igual al del levante anterior. Si es notoriamente mayor, la poda se convierte en una actividad superflua desde el punto de vista cualitativo y en un costo sin ninguna posibilidad de justificarse en el tiempo (Meneses y Velazco, 1992).

Por otro lado, se debe poner énfasis en obtener un pequeño cilindro con defectos y no la compensación de un cilindro defectuoso mayor con un turno más largo (Knowles et.al., 1987; Todoroki, 2003). Por lo tanto, la estimación del DMSM es de importancia fundamental para determinar el momento exacto de realizar los distintos levantes de poda. Para su estimación existen distintas metodologías propuestas por diferentes autores (Knowles et.al., 1987; Fassola et al., 1999; Andenmatten et al., 2003; Chauchard. 2005).

\section{OBJETIVOS}

Estudiar el efecto de la aplicación de diferentes intensidades de poda sobre el crecimiento y la formación de brotes epicórmicos en plantaciones de Populus.

Determinar el momento de realización de cada levante de poda para mantener un diámetro del cilindro con defectos constante a lo largo del fuste podado.

\section{MATERIAL Y MÉTODO}

\section{Área de Estudio}

El estudio abarca parte del valle medio y alto del río Negro y parte de los valles de los ríos Neuquén y Colorado.

\section{Intensidad de poda}

Dentro de este área se realizó la instalación de dos ensayos de intensidad de poda, uno en Añelo, provincia de Neuquén, en una plantación de Populus $x$ canadensis 'I 214' y otro en Lamarque, provincia de Río Negro, donde la especie plantada es Populus $x$ canadensis 'Conti 12'. Ambas plantaciones corresponden a sistemas silvopastoriles, están localizadas en sitios de 
productividad media y tenían 3 años de edad al momento de la instalación de los ensayos (año 2012) (Cuadro $N^{\circ} 1$ ).

Cuadro $\mathrm{N}^{\circ} 1$

DESCRIPCIÓN DE LOS DOS SITIOS

\begin{tabular}{|c|c|c|c|c|c|c|c|}
\hline \multirow[b]{2}{*}{ Localidad } & \multirow{2}{*}{$\begin{array}{c}\text { Distanc. } \\
\text { (m) }\end{array}$} & \multirow[b]{2}{*}{ Especie } & \multirow[b]{2}{*}{ Latitud S } & \multirow[b]{2}{*}{ Longitud 0} & \multirow{2}{*}{$\begin{array}{l}\text { Altitud } \\
\text { (msnm) }\end{array}$} & \multicolumn{2}{|r|}{ Suelo } \\
\hline & & & & & & $\begin{array}{l}\text { Prof. } \\
\text { (m) }\end{array}$ & Textura \\
\hline Lamarque & $3 \times 8$ & Conti 12 & $39^{\circ} 30^{\prime} 28,4^{\prime \prime}$ & $65^{\circ} 41^{\prime} 59,4^{\prime \prime}$ & 123 & 1 & Areno franco \\
\hline Añelo & $3 \times 8$ & | - 214 & $38^{\circ} 31^{\prime} 13,1^{\prime \prime}$ & $69^{\circ} 01^{\prime} 56,9^{\prime \prime}$ & 438 & 1 & Franco arenoso \\
\hline
\end{tabular}

\section{Diseño Experimental}

El diseño del ensayo de Añelo fue de bloques completos al azar, debido a que se observó una diferencia en los crecimientos en altura, en los diferentes bloques, por diferencias en la calidad de sitio. El ensayo de Lamarque fue completamente aleatorizado. Las podas se realizaron en agosto con tijerón y, en el caso de ramas muy gruesas, con serrucho. Se realizaron 3 repeticiones por tratamiento, siendo en total 12 parcelas por ensayo. Cada parcela estaba formada por una parcela de medición interior de 16 árboles y un borde de aproximadamente $8 \mathrm{~m}$.

Los tratamientos aplicados consistieron en distintas intensidades de poda:

Poda fuerte (Pf):

Se podó un $75 \%$ de la altura total de cada árbol, dejando un $25 \%$ de copa verde.

Poda intermedia (Pi): $\quad$ Se podó hasta un $50 \%$ de la altura, dejando un $50 \%$ de copa verde.

Poda suave (Ps): $\quad$ Se podó hasta un $25 \%$ de la altura total, dejando un $75 \%$ de copa verde.

Testigo (T): $\quad$ Sin poda $(100 \%$ de copa).

Estos ensayos fueron objeto de podas, extracción de brotes y medición todos los años desde su instalación. La primera medición se realizó al momento de instalar el ensayo, posteriormente se realizaron tres mediciones, una por año, entre mayo y fines de agosto. A cada árbol de la parcela se le midió:

DAP $(\mathrm{cm})$ : Diámetro medido a 1,30 $\mathrm{m}$ desde el suelo (con cinta diamétrica).

Altura total $(\mathrm{HT})(\mathrm{m})$ : Altura total de cada árbol (con vara graduada).

Altura de poda (HPOD) $(\mathrm{m})$ : Altura a la que se encuentra la primera rama verde luego de la poda (con vara graduada). Se midió luego de cada levante de poda.

Brotes epicórmicos: Se contó el número de brotes epicórmicos sobre el fuste podado, antes de extraerlos cada año.

Los levantes de poda anuales se realizaron manteniendo las intensidades de poda y hasta llegar a una altura total de poda de $6 \mathrm{~m}$. Las parcelas con intensidades de poda de 50 y $75 \%$ ya llegaron a esa altura, en cambio las podadas al $25 \%$ se deben seguir podando. 
Posteriormente, con la información obtenida de los árboles muestra, se evaluó el efecto de la intensidad de poda sobre el crecimiento en DAP y altura y sobre la cantidad de brotes epicórmicos. Para evaluar si las diferencias entre tratamientos eran significativas desde el punto de vista estadístico, se realizó un análisis de varianza y para la comparación entre medias de los distintos tratamientos, el Test de Tukey.

\section{Oportunidad de Cada Levante de Poda}

En el caso de los álamos el diámetro máximo sobre muñón (DMSM) no es notorio, por lo que se optó por utilizar como variable indicadora al diámetro en la base del fuste (DBF) para la primera poda y, para los levantes siguientes, el diámetro en la base de la copa viva (DBCV).

La metodología empleada fue la tradicional, que consiste en relacionar estas variables con otras variables independientes de fácil medición. Las variables se relacionan a través de regresiones múltiples, ajustando funciones independientes para la poda baja y para los levantes de poda posteriores.

Las variables analizadas como variables dependientes, para determinar el momento de poda, fueron para la primera poda el DBF y para los levantes posteriores el DBCV. Las variables a introducir como variables independientes fueron el diámetro a la altura del pecho (DAP), la altura total $(\mathrm{HT})$, el diámetro de la rama viva más gruesa del primer verticilo (DRV) y la altura de poda (HPOD).

La base de datos utilizada estuvo formada por los árboles de los ensayos de intensidad de poda y de una red de parcelas permanentes instaladas en el área en plantaciones podadas. Este análisis se realizó para tres cultivares de Populus $x$ canadensis ('Conti 12', 'I-214' y 'Guardi') (Cuadros $\mathrm{N}^{\circ} 2$ y No 3 ).

\section{Cuadro $\mathrm{N}^{\circ} 2$}

ESTADÍSTICOS DESCRIPTIVOS DE LA BASE DE DATOS EMPLEADA PARA EL AJUSTE DE LOS MODELOS PARA ESTIMAR EL DIÁMETRO EN LA BASE DEL FUSTE (DBF), PARA LAS TRES ESPECIES ANALIZADAS (PRIMER LEVANTE)

\begin{tabular}{|c|c|c|c|c|c|c|}
\hline \multicolumn{7}{|c|}{\begin{tabular}{|l} 
'Conti 12' \\
\end{tabular}} \\
\hline $\mathbf{n}$ & Variable & Media & Máximo & Mínimo & Varianza & Desv. Est. \\
\hline \multirow{5}{*}{282} & $\mathrm{DBF}(\mathrm{m})$ & 8,4 & 18,3 & 2,7 & 7,7 & 2,8 \\
\hline & $\mathrm{DAP}(\mathrm{cm})$ & 6,2 & 14,3 & 2,0 & 4,8 & 2,2 \\
\hline & DBCV $(\mathrm{cm})$ & 6,1 & 13,8 & 2,0 & 5,3 & 2,3 \\
\hline & $\mathrm{HT}(\mathrm{m})$ & 6,3 & 10,8 & 3,8 & 2,4 & 1,6 \\
\hline & $\mathrm{DRV}(\mathrm{mm})$ & 16,9 & 30,0 & 5,0 & 19,3 & 4,4 \\
\hline \multicolumn{7}{|c|}{ 'I-214' } \\
\hline \multirow{5}{*}{178} & $\operatorname{DBF}(\mathrm{m})$ & 10,0 & 19,3 & 3,2 & 16,3 & 4,0 \\
\hline & $\operatorname{DAP}(\mathrm{cm})$ & 6,7 & 15,4 & 1,7 & 16,0 & 4,0 \\
\hline & DBCV $(\mathrm{cm})$ & 7,6 & 14,5 & 2,0 & 9,8 & 3,1 \\
\hline & $\mathrm{HT}(\mathrm{m})$ & 6,6 & 16,0 & 2,7 & 19,9 & 4,5 \\
\hline & $\mathrm{DRV}(\mathrm{mm})$ & 26,1 & 62,0 & 7,0 & 86,2 & 9,3 \\
\hline \multicolumn{7}{|c|}{ 'Guardi' } \\
\hline \multirow{5}{*}{124} & $\mathrm{DBF}(\mathrm{m})$ & 12,1 & 27,0 & 3,5 & 34,9 & 5,9 \\
\hline & $\mathrm{DAP}(\mathrm{cm})$ & 9,2 & 21,1 & 1,6 & 25,1 & 5,0 \\
\hline & $\mathrm{DBCV}(\mathrm{cm})$ & 5,9 & 11,3 & 2,2 & 5,5 & 2,4 \\
\hline & $\mathrm{HT}(\mathrm{m})$ & 8,2 & 16,0 & 2,4 & 14,9 & 3,9 \\
\hline & $\mathrm{DRV}(\mathrm{mm})$ & - & - & - & - & - \\
\hline
\end{tabular}


Cuadro $\mathrm{N}^{\circ} 3$

ESTADÍSTICOS DESCRIPTIVOS DE LA BASE DE DATOS EMPLEADA PARA EL AJUSTE DE LOS MODELOS PARA ESTIMAR EL DBCV PARA LAS TRES ESPECIES ANALIZADAS (LEVANTES POSTERIORES)

\begin{tabular}{|c|c|c|c|c|c|c|}
\hline \multicolumn{7}{|c|}{${ }^{\circ}$ Conti 12' } \\
\hline$n$ & Variable & Media & Máximo & Mínimo & Varianza & Desv. Est. \\
\hline \multirow{3}{*}{354} & $\operatorname{DAP}(\mathrm{cm})$ & 11,1 & 19,6 & 3,2 & 12,40 & 3,52 \\
\hline & $\mathrm{DBCV}(\mathrm{cm})$ & 9,8 & 19,1 & 3,0 & 11,48 & 3,39 \\
\hline & $\operatorname{HPOD}(\mathrm{m})$ & 2,5 & 5,6 & 1,2 & 0,70 & 0,84 \\
\hline \multicolumn{7}{|c|}{ 'I-214' } \\
\hline \multirow{3}{*}{293} & $\mathrm{DAP}(\mathrm{cm})$ & 12,1 & 19,4 & 4,5 & 9,75 & 3,12 \\
\hline & $\mathrm{DBCV}(\mathrm{cm})$ & 10,7 & 21,0 & 4,0 & 9,25 & 3,04 \\
\hline & $\operatorname{HPOD}(\mathrm{m})$ & 2,5 & 4,6 & 0,9 & 0,92 & 0,96 \\
\hline \multicolumn{7}{|c|}{ 'Guardi' } \\
\hline \multirow{3}{*}{84} & $\mathrm{DAP}(\mathrm{cm})$ & 10,4 & 18,4 & 1,8 & 19,94 & 4,47 \\
\hline & $\mathrm{DBCV}(\mathrm{cm})$ & 9,1 & 16,4 & 1,8 & 11,60 & 3,41 \\
\hline & $\operatorname{HPOD}(\mathrm{m})$ & 2,1 & 2,9 & 1,1 & 0,12 & 0,35 \\
\hline
\end{tabular}

Las variables explicatorias se fueron incorporando en el modelo de regresión múltiple utilizando la técnica paso a paso. La evaluación de los modelos se realizó con el Coeficiente de Determinación $\left(\mathrm{R}^{2}\right)$ ajustado por el número de parámetros del modelo. Se realizó un análisis visual del gráfico de residuos para detectar problemas de heterocedasticidad y autocorrelación. Por último, ambos modelos fueron validados mediante medidas de error (REMC) y sesgo (DIFA). Mediante la división por la media de los valores observados y su multiplicación por 100, se obtienen los valores porcentuales (REMC\% y DIFA\%).

La base de datos utilizada para la validación de los modelos de 'I-214' y 'Guardi', fue la misma base de datos empleada para el ajuste de los mismos, debido a la menor cantidad de datos obtenidos. Para la validación del modelo de 'Conti 12', se empleó una base de datos independiente formada por la información de 77 árboles.

Raíz del error medio cuadrático

$$
R E M C=\sqrt{\sum(O-E)^{2} / n}
$$

Diferencia Agregada

$$
D I F A=\sum(O-E) / n
$$

Donde: O: observado; E: estimado; $n$ : $n^{\circ}$ de observaciones

\section{RESULTADOS Y DISCUSIÓN}

\section{Relación entre la Intensidad de Poda y el Crecimiento en Diámetro y Altura}

En cuanto al crecimiento en diámetro transcurridos tres años se observa, en ambos ensayos, una disminución del mismo a medida que aumenta la intensidad de poda. Esto es más notorio en el 'I-214' (Figura $N^{\circ} 1$ ). Sin embargo, el análisis estadístico, no muestra diferencias significativas para 'I-214' $(p=0,10)$ ni para 'Conti 12' $(p=0,22)$ considerando el crecimiento medio de los tres años. Durante los años anteriores las diferencias fueron significativas entre la poda fuerte y el testigo en 'I-214' $(p=0,05)$ y entre la poda fuerte y la poda suave y el testigo en 'Conti 12' $(p=0,02)$. 


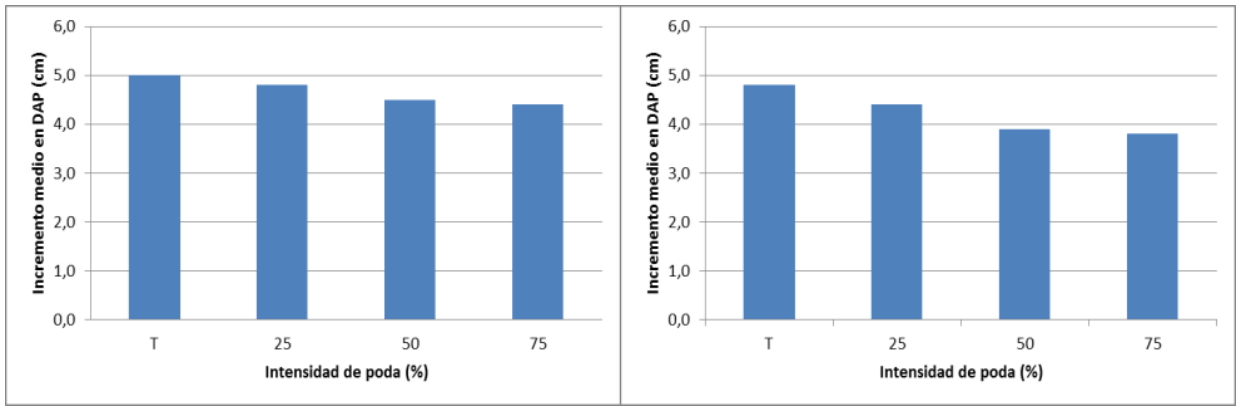

Figura $\mathrm{N}^{\circ} 1$

INCREMENTO MEDIO EN DAP DE Populus $x$ canadensis 'CONTI 12' (IZQUIERDA) Y Populus $x$ canadensis 'I-214’ (DERECHA), PARA LAS TRES INTENSIDADES DE PODA Y EL TESTIGO, LUEGO DE TRANSCURRIDOS TRES AÑOS DE INSTALADOS LOS ENSAYOS

En cuanto al crecimiento en altura, luego de transcurridos tres años, no se observaron diferencias importantes por efecto de la intensidad de poda en 'I-214'.

En cambió en 'Conti 12' pareciera haber una tendencia de disminución de crecimiento en altura con la intensidad de poda, aunque las diferencias son pequeñas (Figura $\mathrm{N}^{\circ} 2$ ).

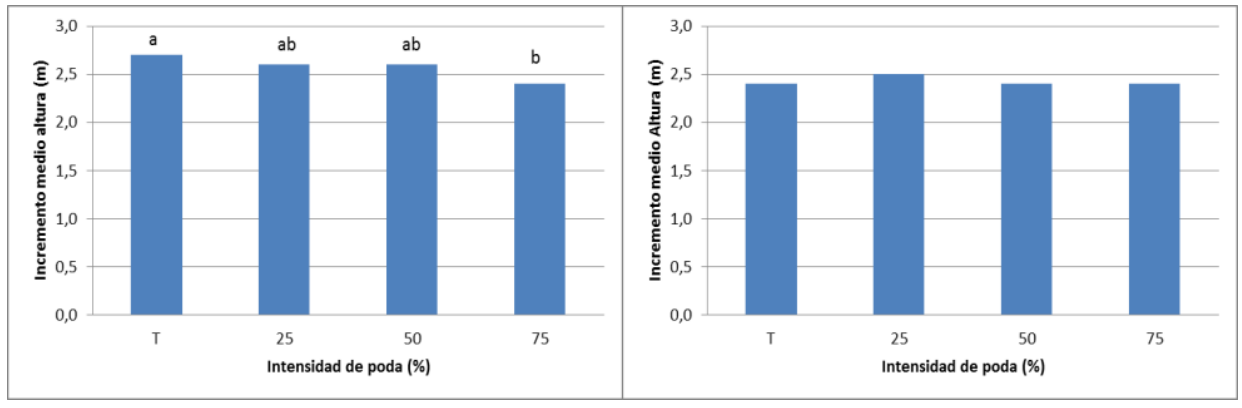

Figura $\mathrm{N}^{\circ} 2$

INCREMENTO MEDIO EN ALTURA DE Populus $x$ canadensis 'CONTI 12' (IZQUIERDA) Y Populus $x$ canadensis 'I-214’ (DERECHA), PARA LAS TRES INTENSIDADES DE PODA Y EL TESTIGO, LUEGO DE TRANSCURRIDOS TRES AÑOS DE INSTALADOS LOS ENSAYOS

Realizado el análisis de varianza, las diferencias observadas no resultaron significativas para 'I-214' $(p=0,33)$. En cambio para 'Conti 12', al tercer año, las diferencias son significativas entre el testigo y la poda fuerte $(p=0,059)$. Esto no se había observado en los años anteriores.

\section{Efecto de la Intensidad de Poda sobre la Frecuencia de Brotes Epicórmicos}

En el Cuadro $\mathrm{N}^{\circ} 4$ se presentan los resultados obtenidos en frecuencia de brotes epicórmicos para cada intensidad de poda, a lo largo de los años. Si bien se observa que hay un mayor número de brotes a medida que incrementa la intensidad de poda, también se observa una disminución del número de los mismos, en todos los tratamientos, a medida que pasan los años. 
FRECUENCIA DE BROTES EPICÓRMICOS PARA CADA TRATAMIENTO DE PODA DE CADA ENSAYO Y PARA LOS TRES AÑOS EVALUADOS

\begin{tabular}{|c|c|c|c|c|}
\hline \multirow{2}{*}{$\begin{array}{l}\text { Tratamiento } \\
\% \text { poda }\end{array}$} & \multicolumn{4}{|c|}{ 'Conti 12' - número de brotes epicórmicos } \\
\hline & 2012 & 2013 & 2014 & 2015 \\
\hline $\begin{array}{l}25 \\
50 \\
75\end{array}$ & $\begin{array}{c}3 \\
6 \\
10 \\
\end{array}$ & $\begin{array}{c}3 \\
10 \\
15\end{array}$ & & $\begin{array}{c}1 \\
3 \\
12\end{array}$ \\
\hline \multirow{2}{*}{$\begin{array}{l}\text { Tratamiento } \\
\% \text { poda }\end{array}$} & \multicolumn{4}{|c|}{ 'I-214’ - número de brotes epicórmicos } \\
\hline & 2012 & 2013 & 2014 & 2015 \\
\hline $\begin{array}{l}25 \\
50 \\
75\end{array}$ & $\begin{array}{c}4 \\
7 \\
34\end{array}$ & $\begin{array}{c}7 \\
12 \\
10\end{array}$ & $\begin{array}{l}1 \\
2 \\
1\end{array}$ & $\begin{array}{l}1 \\
3 \\
7\end{array}$ \\
\hline
\end{tabular}

(En ‘Conti 12' la información faltante, en el año 2014, se debe a que hubo daños por ganado)

\section{Efecto de la Intensidad de Poda sobre el Diámetro del Cilindro con Defectos}

Cuando el objetivo de una plantación es producir madera de calidad, un aspecto fundamental es que el diámetro del cilindro con defectos se mantenga constante a lo largo de todo el fuste podado.

No se puede medir el diámetro de ese cilindro en un árbol en pie, lo que sí se puede hacer, es controlar que se mantenga constante a través del DBF y los DBCV. Es decir, que en una poda bien realizada, el DBCV al realizar el segundo levante de poda debe ser igual al DBF al momento de realizar el primer levante de poda y el DBCV al realizar el tercer levante de poda debe ser igual al DBF y al DBCV del segundo levante. Así se asegura un diámetro del cilindro con defectos (DCD) más o menos constante a lo largo del fuste podado.

Realizando un levante por año, tanto para 'Conti 12' como para 'I-214', se observó que si se realiza la primera poda cuando los árboles tienen un DBF de $8 \mathrm{~cm}$ y se poda con una intensidad del $25 \%$, el valor de estos diámetros de referencia y por lo tanto del DCD, va a aumentar en forma importante (aproximadamente entre 6 y $8 \mathrm{~cm}$ luego de los tres levantes).

Con esta intensidad de poda, el $100 \%$ de los árboles incrementaba el valor de estos diámetros en los distintos levantes de poda. Es decir se perderían los beneficios de haber realizado una primera poda en el momento adecuado.

Si se poda al $50 \%$, las diferencias son menores, sobre todo en 'I-214', aunque también hay un incremento de entre 3 y $4 \mathrm{~cm}$. Esto ocurría en aproximadamente el $60 \%$ de los individuos. Esto se podría solucionar realizando dos levantes de poda por año, uno a fines de invierno y otro a fines de verano.

Podando al $75 \%$ se logra, para ambas especies, mantener el valor de estos diámetros constante a lo largo del fuste podado. En promedio entre las dos especies solo un $7 \%$ de los árboles se pasaría en el valor del DBCV aplicando esta intensidad de poda.

En la Figura $N^{\circ} 3$ se puede observar el comportamiento de estas variables en 'I 214 '. 


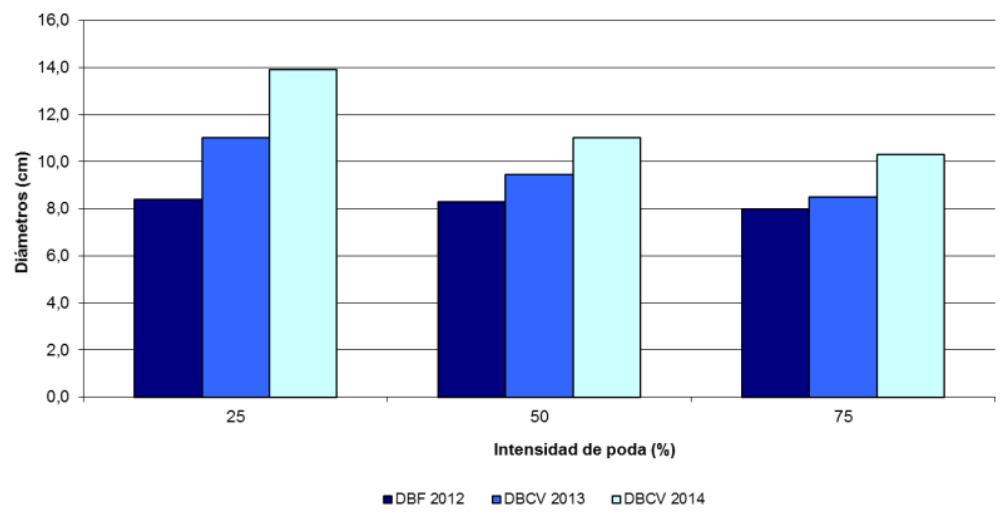

Figura $N^{\circ} 3$

EVOLUCIÓN DE LOS DIÁMETROS (DBF Y DBCV) PARA LAS DISTINTAS INTENSIDADES DE PODA Y TENIENDO EN CUENTA LOS 3 LEVANTES EN 'I-214'.

\section{Cantidad de Levantes de Poda para Llegar a la Altura de Poda Objetivo}

Si se establece como objetivo de poda, lograr un fuste de $6 \mathrm{~m}$ libre de ramas y teniendo en cuenta los crecimientos obtenidos para ambas especies en los sitios de ensayo, se observa en el Cuadro $\mathrm{N}^{\circ} 5$ que con una poda del $75 \%$ se llega a los $6 \mathrm{~m}$ en tres levantes realizados en tres años consecutivos. Con la intensidad de poda del $50 \%$ se deberían realizar cuatro levantes y con la intensidad de poda de $25 \%$ se deberían realizar entre 7 y 8 levantes en total. En el caso de la poda del $50 \%$ se podría realizar más fuerte el tercer levante para llegar hasta los $6 \mathrm{~m}$ de fuste podado.

\section{Cuadro $\mathrm{N}^{\circ} 5$ \\ ALTURA DE PODA LOGRADA EN CADA LEVANTE DE PODA, PARA CADA INTENSIDAD EN ‘CONTI 12’ E 'I-214’}

\begin{tabular}{|c|c|c|c|}
\hline \multirow{2}{*}{$\begin{array}{c}\text { Tratamiento } \\
\text { de Poda } \\
(\%)\end{array}$} & \multicolumn{3}{|c|}{ 'Conti 12' - altura de poda } \\
\cline { 2 - 4 } & Primer Levante & Segundo Levante & Tercer Levante \\
\cline { 2 - 4 } & \multicolumn{3}{|c|}{$(\mathrm{m})$} \\
\hline 25 & 1,5 & 2,0 & 3,9 \\
50 & 2,5 & 3,7 & 5,0 \\
75 & 3,3 & 4,8 & 5,8 \\
\hline \multicolumn{4}{|c|}{ 'I-214' - altura de poda } \\
\hline 25 & 1,3 & 2,0 & 3,7 \\
50 & 1,8 & 3,4 & 5,8 \\
75 & 3,8 & 4,5 & 5,8 \\
\hline
\end{tabular}

\section{Determinación del Momento de Cada Levante de Poda}

\section{Primer Levante}

De todas las variables analizadas, el DAP fue la que mejor explicó la variación del DBF para las tres especies, presentando los valores más altos de $R^{2}$ y una buena distribución de los residuos. En la Figura $\mathrm{N}^{\circ} 4$ se presenta, como ejemplo, la relación entre el DAP y el DBF y los gráficos de residuales para 'Conti 12'. 

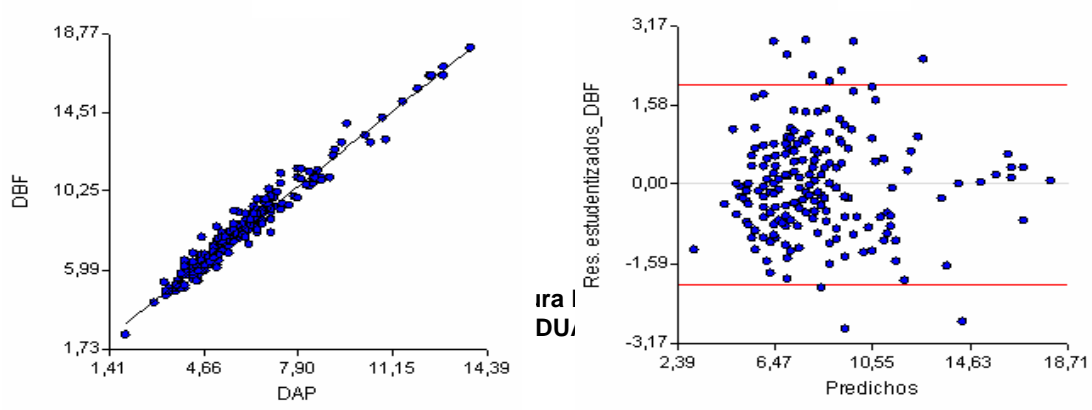

El modelo obtenido para estimar el DBF a partir del DAP tiene la siguiente expresión:

$D B F=a+b * D A P$

Los resultados del ajuste del mismo para cada una de las especies analizadas se presentan en el Cuadro $\mathrm{N}^{\circ} 6$.

Cuadro $\mathrm{N}^{\circ} 6$

COEFICIENTES OBTENIDOS EN EL AJUSTE, $R^{2}$ Y P-VALOR DEL MODELO PARA ESTIMAR EL DBF

\begin{tabular}{|l|c|c|c|c|}
\hline Especie & a & b & $\mathbf{R}^{2}$ & p-valor \\
\hline P. x canadensis 'Conti 12' & 0,62 & 1,26 & 0,97 & $<0,0001$ \\
P. x canadensis 'I-214' & 3,38 & 0,99 & 0,97 & $<0,0001$ \\
P. x canadensis 'Guardi' & 1,32 & 1,17 & 0,98 & $<0,0001$ \\
\hline
\end{tabular}

Luego de ajustados, estos modelos fueron validados mediante medidas de error (REMC\%) y sesgo (DIFA\%) (Cuadro $\mathrm{N}^{\circ} 7$ ). Como se puede observar, para las tres especies, se observan muy buenos comportamientos de los modelos, con muy bajos valores de error y prácticamente insesgados en sus estimaciones. Los modelos correspondientes a 'Conti 12' y 'Guardi' producen una leve sobrestimación del DBF, mientras que en el caso del 'I-214' presenta una pequeña subestimación.

\section{Cuadro $\mathrm{N}^{\circ} 7$ \\ MEDIDAS DE ERROR (RECM\%) Y SESGO (DIFA\%) DE LOS MODELOS AJUSTADOS PARA ESTIMAR DBF}

\begin{tabular}{|l|c|c|}
\hline Especie & $\begin{array}{c}\text { RECM } \\
(\%)\end{array}$ & $\begin{array}{c}\text { DIFA } \\
\text { (\%) }\end{array}$ \\
\hline P. $x$ canadensis 'Conti 12' & 3,57 & $-0,25$ \\
P. x canadensis 'I-214' & 5,31 & 0,25 \\
P. x canadensis 'Guardi' & 6,15 & $-0,11$ \\
\hline
\end{tabular}

Considerando que las debobinadoras existentes trabajan hasta un diámetro de $10 \mathrm{~cm}$ y haciendo el supuesto de que la cicatrización implique unos $2 \mathrm{~cm}$ en el diámetro, para lograr un DCD de $10 \mathrm{~cm}$ promedio, según estos modelos, se deberían realizar las podas cuando el DBF tiene un valor de $8 \mathrm{~cm}$ y esto ocurre cuando el DAP de los árboles dominantes del rodal alcancen los $4 \mathrm{~cm}$ para 'I 214' y de $5 \mathrm{~cm}$ para 'Conti 12 ' y 'Guardi'. 
Además, estos modelos están indicando que por cada centímetro de aumento en el DAP, el DBF aumenta $1,26 \mathrm{~cm}$ en 'Conti-12', aproximadamente $1 \mathrm{~cm}$ en 'I-214' y 1,17 cm en 'Guardi'. Esto permite cuantificar en cuanto se incrementa el DBF y, por lo tanto, el DCD por un atraso en el momento de realizar la primera poda y en cuanto se debe alargar el turno de corta para obtener el mismo volumen de madera libre de nudos. Por ejemplo, si se considera los crecimientos en DAP obtenidos en el ensayo de intensidad de poda de 'Conti 12' (Lamarque, Río Negro) que fueron de aproximadamente $5 \mathrm{~cm}$ por año, se tendría que un atraso de un año en la primera poda, significaría un incremento en el DBF de $6,3 \mathrm{~cm}$.

\section{- $\quad$ Levantes Posteriores}

Para determinar el momento de la segunda poda se evaluó la relación del DAP, la altura total (HT) y la altura de poda (HPOD) con el diámetro en la base de la copa viva (DBCV), mediante regresión múltiple. Se observó que, de estas variables, las que explican mejor el comportamiento del DBCV para las tres especies analizadas son el DAP y la altura de poda (HPOD) del levante anterior. El modelo obtenido para estimar el DBCV a partir del DAP y la HPOD, tiene la siguiente expresión:

$$
D B F=a+b * D A P+c * H P O D
$$

Los resultados del ajuste del mismo para cada una de las especies analizadas se presentan en el Cuadro $\mathrm{N}^{\circ} 8$.

Cuadro $\mathrm{N}^{\circ} 8$

COEFICIENTES OBTENIDOS EN AJUSTE, $\mathbf{R}^{2}$ Y P-VALOR DE MODELO OBTENIDO PARA ESTIMAR DBCV

\begin{tabular}{|l|c|c|c|c|c|}
\hline Especie & a & b & C & $\mathrm{R}^{2}$ & p-valor \\
\hline P. x canadensis 'Conti 12' & 2,07 & 0,94 & 1,08 & 0,97 & $<0,0001$ \\
P. x canadensis 'I-214' & 3,02 & 0,93 & 1,47 & 0,92 & $<0,0001$ \\
P.x canadensis 'Guardi' & 1,90 & 0,77 & 0,39 & 0,96 & $<0,0001$ \\
\hline
\end{tabular}

Luego de ajustados, estos modelos fueron validados mediante medidas de error (REMC\%) y sesgo (DIFA\%) al igual que como se hizo para evaluar los modelos ajustados para el primer levante de poda (Cuadro $\mathrm{N}^{\circ}$ 9).

Cuadro $\mathrm{N}^{\circ} 9$

MEDIDAS DE ERROR (ECM\%) Y SESGO (DIFA\%) DE LOS MODELOS PARA ESTIMAR DBCV

\begin{tabular}{|l|c|c|c|}
\hline Especie & N & $\begin{array}{c}\text { RECM } \\
(\%)\end{array}$ & $\begin{array}{c}\text { DIFA } \\
\text { (\%) }\end{array}$ \\
\hline P. $x$ canadensis 'I-214' & 53 & 4,74 & $-0,53$ \\
P. x canadensis 'Conti 12' & 103 & 3,13 & 0,66 \\
P. $x$ canadensis 'Guardi' & 33 & 6,86 & 4,21 \\
\hline
\end{tabular}

$\mathrm{N}$ indica el número de datos de la base utilizada para la validación de los modelos.

\section{CONCLUSIONES}

En contra de lo esperado, no se observaron diferencias en el crecimiento en diámetro para las distintas intensidades de poda aplicadas luego de transcurridos tres años. Tampoco se observaron diferencias en el crecimiento en altura.

En 'I- 214', hubo diferencias significativas en la cantidad de brotes epicórmicos formados luego del primer levante de poda. Los árboles podados hasta un $75 \%$ de su altura, tuvieron una 
mayor aparición de brotes que los podados hasta el $25 \%$ y el $50 \%$ de la altura total. Sin embargo, en los años siguientes, las diferencias son menores y terminan siendo no significativas al tercer año, donde el número de brotes disminuye en forma importante.

En 'Conti 12', se encontraron diferencias en cantidad de brotes según la intensidad de poda empleada. En cuanto a la cantidad de brotes, se pudo ver que, en los ejemplares podados al $75 \%$ de su altura, hubo una mayor incidencia de rebrotes que en los otros dos tratamientos, coincidiendo esto con 'I-214'. Al segundo año las diferencias se dan entre la poda más suave (25 $\%$ ) y las otras dos intensidades de poda (50 y $75 \%$ ).

En cuanto al comportamiento del diámetro del cilindro con defectos, realizando levantes anuales, el mejor resultado se obtiene con una poda del $75 \%$. Si se realizan levantes anuales del $25 \%$ el DBCV y por lo tanto el DCD van aumentar entre 6 y $8 \mathrm{~cm}$ del primero al tercer levante. La poda de $50 \%$ está en una situación intermedia y se podrían mejorar los resultados, realizando dos levantes anuales.

Si se establece como objetivo de poda lograr un fuste de $6 \mathrm{~m}$ libre de ramas y teniendo en cuenta los crecimientos obtenidos para ambas especies en estos sitios, se observó que con una poda del $75 \%$, se deben realizar tres levantes en tres años consecutivos. Con la intensidad de poda del $50 \%$ se deberían realizar cuatro levantes, y con la intensidad de poda de $25 \%$ se deberían realizar entre 7 y 8 levantes.

EI DAP presenta una fuerte relación con el diámetro en la base del fuste (DBF), en la primera poda, para las tres especies. A partir de esta variable se puede determinar en forma precisa el momento de realizar el primer levante de poda. La relación encontrada indica que para obtener un DBF de $7 \mathrm{~cm}$, se debe realizar la primera poda cuando los árboles más grandes del rodal tienen un DAP de alrededor de $5 \mathrm{~cm}$ para 'Conti-12' y 'Guardi' y de $4 \mathrm{~cm}$ para 'I-214'.

Teniendo en cuenta los crecimientos observados en las plantaciones estudiadas, esta relación indica que un atraso de un año en realizar el primer levante de poda produce aumentos importantes en el DBF y, por lo tanto, pérdidas en la producción de madera de calidad. Estos

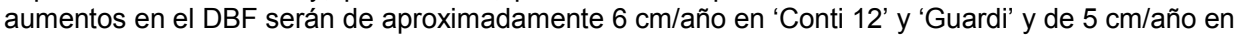
'I-214'.

Para los levantes posteriores se encontró, que las variables que permiten determinar el momento exacto de realizarlos son el DAP y la altura de poda del levante anterior. Además las relaciones encontradas, para las tres especies, muestran que por cada centímetro que aumente el DAP, el diámetro máximo sobre muñón aumentará aproximadamente $0,8 \mathrm{~cm}$ en 'Guardi' y 0,94 cm en 'I-214' y 'Conti 12' y que cuanto menor sea la altura de poda más rápido se va a tener que volver a podar para mantener constante el cilindro con defectos central.

En base a los resultados obtenidos hasta el momento se recomienda podar entre el $50 \%$ y el $75 \%$ de la altura total de cada árbol en cada levante de poda. Podar por debajo del $50 \%$ de la copa viva implicaría un mayor número de entradas al monte para alcanzar la misma altura de poda; un menor número de rebrotes pero, solo al primer año, luego disminuye el número de brotes chupones en las podas más fuertes; y similares crecimientos en diámetro y altura de los árboles que en las podas más fuertes. Se debe evaluar aún, en base a información ya obtenida, cómo es el efecto sobre el diámetro del cilindro con defectos (DCD).

\section{REFERENCIAS}

Andenmatten, E.; Fassola, H.; Letourneau, F.; Ferrere, P. y Crechi, E., 2003. Predicción de diámetro sobre muñones en Pinus taeda L. origen marion mediante curvas de perfil de fuste. INTA. RIA 31(3):103-118. Argentina.

Casaubon, E., 2004. Poda de álamos en el Delta del Paraná. Boletín Extensión no 16. INTA E.E.A. Delta del Paraná. $4 p$. 
Chauchard, L., 2005. Modelo de poda para pino radiata. Nota Técnica. Diputación Foral de Guipúzcoa. 39p.

Costas, R.; Mac Donagh, P.; Weber, E.; Irchick, P. y Figueredo, S., 2003. Efectos de la densidad de plantación y tratamientos de poda sobre la producción de Pinus taeda L. a los 7 años de edad. Décimas Jornadas Técnicas Forestales y Ambientales. Facultad de Cs. Forestales - UNAM - EEA Monte Carlo - INTA. Eldorado, Misiones. $10 \mathrm{p}$.

Davel, M., 2005. Poda en plantaciones de pino oregón en la Patagonia. Publicado en Actas del 3er Congreso Forestal Argentino y Latinoamericano. Corrientes. 6-9 de septiembre de 2005.

Fassola, H.; Rodríguez, F.; Allegranza, D.; Hernández, A.; Ferrere, P.; Durán, M. y Reboratti, H., 1999. Resultados iniciales de tratamientos silvícolas directos en Pinus taeda origen Marion, en el NE de Corrientes. Informe Técnico №22. INTA EEA Montecarlo, Misiones. 9 p.

García, J. y Serventi, N., 2012. Mercado de la madera industrializada en Norpatagonia. http://patagoniavallesirrigados.blogspot.com.ar/2012/07/mercado-de-la-madera-industrializada-en.html

Knowles, R.; West, G. y Koehler, A., 1987. Predicting "diameter over stubs" in pruned stands of Radiata Pine. Ministry of Forestry, Forest Research Institute, Rotorua, New Zealand. Bulletin № 12. 24pp.

Kurtz, V. y Ferruchi, R., 2000. La poda como parte de la estrategia para la obtención de madera de calidad. XV Jornadas Forestales de Entre Ríos. 23p.

Meneses, M. y Velazco, R., 1992. Comportamiento del diámetro del cilindro con defectos en parcelas sometidas a diversos tratamientos de poda y raleo. Pinus radiata, investigación en Chile. Silvicultura, Manejo y Tecnología. UACH. 109-123.

Rivera, H. y Sobarzo, M., 1992. Efecto de raleo y poda extrema en plantaciones de pino radiata. Pinus radiata, investigación en Chile. Silvicultura, Manejo y Tecnología. UACH. 124 -134.

Serventi, N. y García, J., 2004. Revista SAGPyA Forestal №32: 24-27. Septiembre, 2004. Bs.As. Argentina.

Serventi, N., 2011. Las cortinas forestales en los valles irrigados de norpatagonia. Jornadas de salicáceas 2011. 3ํㅡㄹ Congreso Internacional de Salicáceas en Argentina. Neuquén. 16 al 18 de marzo de 2011.

Shock, C.; Feibert, E. and Eaton, J., 2000. Effect of pruning severity on the annual growth of hybrid Poplar. Malheur Experiment Station, Oregon State. University Ontario.

http://www.cropinfo.net/AnnualReports/2000/popprune2000.htm

Sutton, W. and Crowe, J., 1975. Selective pruning of Radiata Pine. New Zeland Journal Forestry Science. 5(2):171-195.

Todoroki, C., 2003. Importance of maintaining defect cores. New Zealand Journal of Forestry Science 33 (1): 25 -34 .

Valor Agregado, 2005. La industria maderera en cifras. Ed: E.H. Rodrigo. Bs.As. Argentina. p 41-49. 